\title{
GANANCIA GENÉTICA ESPERADA EN Acacia mangium EN LOS CHILES, ZONA NORTE DE COSTA RICA ${ }^{1}$
}

\author{
Benjamín Pavlotzky-Blank ${ }^{2}$,Olman Murillo-Gamboa ${ }^{3}$
}

\section{RESUMEN}

Ganancia genética esperada en Acacia mangium en Los Chiles, zona norte de Costa Rica. Con el objetivo de seleccionar los materiales de mayor crecimiento y calidad de fuste en Acacia mangium, se evaluó un ensayo de progenie de Acacia mangium Willd. conformado por veinticinco familias. El ensayo fue establecido en Los Chiles, zona norte de Costa Rica, en 2006 con evaluaciones en el 2007 y en 2010. Se utilizó material genético seleccionado por la Cooperativa de Conservación y Mejoramiento Genético Forestal "GENFORES", en Costa Rica y Colombia. Cada familia estuvo representada por 48 progenies, plantadas en cuatro parejas distribuidas en forma aleatoria dentro de cada uno de los seis bloques del ensayo. Se evaluó el diámetro a la altura de pecho "DAP", incremento en DAP, adaptabilidad al sitio, número de trozas comerciales, bifurcación, altura de bifurcación, calidad de las primeras cuatro trozas. Se determinó el volumen de madera comercial por árbol y hectárea. Los datos fueron analizados por medio del software SELEGEN de EMBRAPA para obtener los parámetros genéticos. Todos los caracteres registraron valores de heredabilidad media familiar superiores a 0,68. $\mathrm{Si}$ se seleccionaran los dos mejores individuos dentro de las mejores doce familias, se obtendría una ganancia genética del $40,8 \%$ en volumen comercial/ha a los cuatro años de edad. Esta ganancia corresponde a un volumen comercial de 91,65 $\mathrm{m}^{3} / \mathrm{ha}$, a una tasa de $22,9 \mathrm{~m}^{3} / \mathrm{ha} /$ año. Las dos procedencias derivadas de Colombia son significativamente superiores a los demás materiales evaluados. El análisis de correlación genética entre caracteres muestra que la tasa de crecimiento diamétrico se expresa desde temprana edad en esta especie, lo que podría ser utilizado a futuro en una selección a menor edad.

Palabras clave: Mejoramiento genético, procedencias, progenie, correlación genética.

\begin{abstract}
Expected genetic gain in Acacia mangium in Los Chiles, northern of Costa Rica. An Acacia mangium Willd. progeny test conformed by 25 families was evaluated with the objective of selecting the best materials in growth and stem quality. The trial was established in Los Chiles, Northern Costa Rica in 2006, and evaluated in 2007 and in 2010. Genetic materials came from breeding selections obtained by GENFORES, a tree improvement and gene conservation cooperative, in Costa Rica and Colombia. Each family was represented by 48 progenies, split in four pairs randomly distributed within each of the six blocks of the trial. $\mathrm{DBH}, \mathrm{DBH}$ increment, survival rate, number of commercial logs per tree, forking, forking height and $\log$ quality of the first four logs were evaluated. Wood commercial volume per tree and hectare was estimated. Data was analyzed with SELEGEN software from EMBRAPA in order to obtain all genetic parameters for the breeding population. All traits showed family mean heritability values over 0.68 . Genetic gain in commercial volume per hectare was estimated as $40.8 \%$, when selecting as progenitors the two best individuals from the top twelve families at four year old, which corresponds to an expected commercial volume/ ha at this age of $91.65 \mathrm{~m}^{3} / \mathrm{ha}$, based on a growth rate of 22.9 $\mathrm{m}^{3} /$ ha/year. The two columbian provenances are significantly superior to the rest of the evaluated materials. Genetic correlations among traits show diameter growth rate early expressed in this tree species, therefore, there is a potential usage of this trait in early selection thus, shortening future selections.
\end{abstract}

Key words: Tree improvement, provenances, progeny test, genetic correlation.

\footnotetext{
Recibido: 24 de noviembre, 2011. Aceptado: 12 de marzo, 2012. Parte de la Tesis de licenciatura del primer autor, realizada en la Universidad Nacional.

2 Sistema Nacional de Áreas de Conservación, San José, Costa Rica. bpavlotzky@gmail.com

3 Escuela de Ingeniería Forestal, Instituto Tecnológico de Costa Rica, Cartago, Costa Rica. olmuga@yahoo.es
} 


\section{INTRODUCCIÓN}

La acacia (A. mangium) ha sido reconocida por su capacidad de crecimiento y alta producción de biomasa, en suelos degradados, ácidos, de baja fertilidad y poco contenido de materia orgánica (Nirsatmanto et al. 2004, Kim et al. 2008).

El género Acacia, perteneciente a la familia de las Fabaceas, algunas de sus especies han tenido éxito en los programas de reforestación comercial en la zona oriental del trópico asiático: en Vietnam, van Bueren (2004) estimó una superficie de 127000 ha plantadas con híbrido de acacia; en este mismo país (Kim et al. 2008), estimaron 500000 ha plantadas con A. mangium, A auriculiformis y el híbrido de ambas; en Indonesia Nirsatmanto et al. (2004) estimaron 800000 ha de A. mangium. En el trópico asiático esta especie se ha convertido en la opción de plantación que genera la mayor productividad en ciclos cortos de crecimiento.

$\mathrm{La}$ especie fue introducida en Costa Rica en los años 80, como parte de las investigaciones para la producción de leña y energía en Centroamérica (CATIE 1992). A pesar de su gran potencial, es una especie que ha recibido poca atención por parte de los silvicultores nacionales, entre ellos, algunos esfuerzos recientes sobre aspectos de podas y raleos (Guevara y Murillo 2009).

Como parte del manejo exitoso de esta especie en plantaciones comerciales, es necesaria la selección y desarrollo de fuentes de semilla o material propagativo con individuos que muestren superioridad genética en calidad del fuste, tasa de crecimiento y calidad de la madera. Trabajos en mejoramiento genético iniciados en la costa caribe colombiana, reportan estimados de progreso genético de un $30 \%$ en altura comercial, un $60 \%$ en volumen comercial y aproximadamente un 28\% en calidad del fuste (Espitia et al. 2010).

Para seleccionar los mejores individuos con base en sus méritos genéticos, es necesario estimar parámetros genéticos en la población de mejoramiento genético bajo evaluación. Nuevos procedimientos estadísticos avanzados, como modelos mixtos por medio de los métodos de Máxima Verosimilitud Restringida (Restricted Maximum Likelihood, REML) y Mejor Predicción Linear No Sesgada (Best Linear Unbiased Prediction, BLUP) están ahora disponibles en el software desarrollado por Resende en EMBRAPA, Brasil (Resende 2006). La ventaja de estos métodos es que se pueden utilizar también con bases de datos no balanceados.

La investigación tiene el objetivo de determinar diferencias genéticas entre progenies de A. mangium, procedentes de diferente origen geográfico. De esta forma se espera obtener mejor material genético en cuanto a su tasa de crecimiento, calidad y adaptabilidad a los suelos ácidos, pobres y de menor costo económico propios del cantón de Los Chiles, Alajuela (Murillo y Badilla 2004a) proveyendo a diversas empresas reforestadoras locales de un material que les genere mayor beneficio, aportando a la economía nacional, reduciendo desempleo y en armonía con el ambiente.

La investigación forma parte del programa de mejoramiento genético de GENFORES (Cooperativa de mejoramiento genético forestal, Escuela de Ingeniería Forestal, Instituto Tecnológico de Costa Rica) y representa el primer esfuerzo en mejoramiento genético de A. mangium en el país.

\section{MATERIALES Y MÉTODOS}

Durante el año 2006 se estableció un ensayo de progenie de veinticinco familias de A. mangium en el cantón de Los Chiles, provincia de Alajuela, Costa Rica.

Se utilizaron progenies provenientes de árboles plus de Colombia (códigos $\mathrm{K}=$ Kanguroid y $\mathrm{M}=$ Cordoba) y Costa Rica (códigos $\mathrm{P}=$ Parrita y $\mathrm{C}=\mathrm{El}$ Concho, San Carlos), así como lotes mezclados provenientes de República Dominicana (RDom) y Papúa Nueva Guinea. Todas las familias fueron obtenidas de polinización abierta (medios hermanos). El ensayo forma parte del programa de mejoramiento genético de la cooperativa GENFORES con A. mangium.

El diseño experimental del ensayo genético fue el propuesto y empleado por GENFORES (Murillo et al. 2001, Murillo y Badilla 2004b). Consistió en un diseño de seis bloques completos al azar. Dentro de cada uno se distribuyó aleatoriamente cuatro parejas de medios hermanos distantes entre sí, con el objetivo posterior de convertirse en huerto semillero. Los árboles fueron plantados con un distanciamiento de $3 \mathrm{~m} \mathrm{x}$ $3 \mathrm{~m}$. El terreno fue previamente subsolado a $30 \mathrm{~cm}$ de profundidad sobre las líneas de siembra, y posteriormente rastreado.

El área del ensayo pertenece a la zona de vida bosque húmedo tropical transición a perhúmedo. La 
precipitación promedio (mm/año) es de: $2500^{4}-3000^{5}$, el brillo solar (horas/año): $1825^{6}$. La temperatura promedio anual $\left(\mathrm{C}^{\circ}\right)$ : 25,3 y la velocidad máxima del viento $(\mathrm{km} / \mathrm{h})$ es de $31^{7}$. El orden de suelos es Ultisol ${ }^{8}$. La calidad de sitio es Media según las mediciones realizadas y lo reportado por Murillo y Badilla (2006). Los caracteres evaluados a los cuatro años de edad fueron:

- diámetro a 1,3 m (DAP);

- incremento corriente anual (ICA) del DAP en relación con una medición del 2007;

- calidad de las primeras cuatro trozas de 2,5 m de longitud según la metodología propuesta por $\mathrm{Mu}-$ rillo y Badilla (2004b) (Cuadro 1);

- presencia o ausencia de bifurcación (pérdida del eje principal);

- número de troza en que se encuentra la primera bifurcación estimada visualmente (altura de bifurcación);

- mortalidad;

- volumen comercial sin corteza de cada árbol (a una altura comercial hasta los $10 \mathrm{~cm}$ de diámetro o una menor altura si se ha perdido la dominancia apical) calculado con el programa Avalúos Forestales (Murillo y Badilla 2011), que ajusta los datos con base en la calidad de las trozas;

- volumen comercial por hectárea, se obtuvo al multiplicar la mortalidad promedio de cada familia con el volumen comercial por árbol, multiplicado por una densidad inicial de 1111 árboles/ha.

Los valores de calidad de cada troza individual se sumaron de manera ponderada (según valores del Cuadro 1) para obtener un valor de calidad general (Cal) del árbol, que permitiera estimar el porcentaje de madera comercializable.

La calidad se cuantificó en una escala de valor porcentual, donde 100 es la mejor calidad posible y 0 la peor.

El análisis genético de los datos se realizó con el software SELEGEN (Resende 2007), utilizando el modelo 1 (bloques al azar, progenies de medios hermanos, varias plantas por parcela) y el 24 (bloques

\footnotetext{
${ }_{4}$ ECODirecta, 2007.

5 Instituto Meteorológico Nacional, citado por SIRZEE (S.f.:2010)

6 ECODirecta, 2007.

7 Instituto Meteorológico Nacional, citado por SIRZEE (1985:2010).

8 Instituto Geográfico Nacional, citado por SIRZEE (1989:2010).
}

Cuadro 1. Peso económico relativo de cada troza a lo largo del árbol (Espitia et al. 2010).

\begin{tabular}{cccccc}
\hline $\begin{array}{c}\mathbf{N}^{0} \text { de trozas } \\
\text { en el árbol }\end{array}$ & $\begin{array}{c}\text { 1er } \\
\text { troza }\end{array}$ & $\begin{array}{c}\mathbf{2 d a} \\
\text { troza }\end{array}$ & $\begin{array}{c}\text { 3ra } \\
\text { troza }\end{array}$ & $\begin{array}{c}\text { 4ta } \\
\text { troza }\end{array}$ & $\begin{array}{c}5 \text { ta } \\
\text { troza }\end{array}$ \\
\hline 1 & 100 & - & - & - & - \\
2 & 60 & 40 & - & - & - \\
3 & 45 & 33 & 22 & - & - \\
4 & 40 & 30 & 20 & 10 & - \\
5 & 35 & 25 & 20 & 15 & 5 \\
\hline
\end{tabular}

al azar, prueba de poblaciones o procedencias, varias plantas por parcela):

$\begin{array}{ll}\text { Modelo 1: } & y=X r+Z a+W p+e \\ \text { Modelo 24: } & y=X r+Z g+W p+e\end{array}$

Donde " $y$ " es el vector de datos, " $r$ " es el vector de los efectos de la repetición sumados a la media general, "a" es el vector de los efectos genéticos aditivos individuales, "g" es el vector de los efectos genéticos de poblaciones (asumidos como aleatorios), "p" es el vector de los efectos de la parcela, "e" es el vector de errores residuales. Las letras mayúsculas representan las matrices de incidencia para los efectos referidos (Resende 2006).

También se utilizó el modelo 106 (Optimización de la selección en función de la endogamia y del tamaño efectivo poblacional), para seleccionar los mejores dos árboles por familia. Con esto se pretende obtener una ganancia genética alta con la mayor diversidad posible en la población de mejoramiento.

Se realizó el marcaje del raleo aprovechando que en el diseño experimental los árboles fueron plantados en parejas de medios hermanos, aleatorizadas espacialmente dentro de cada bloque. Se seleccionó y eliminó fenotípicamente el peor árbol de cada pareja (subparcela). Se realizó entonces un análisis de datos pre y post raleo.

Se utilizó mediciones del DAP a un año de edad para analizar genéticamente la tasa de incremento entre esta medición y la realizada al cuarto año.

La correlación genética entre caracteres se estimó después de que fueron eliminados los efectos ambientales (ecuación 4), con base en el software SELEGEN (Resende 2007). La significancia de los valores de correlación fue evaluada con la prueba 
estadística de “t” (ecuación 5), según el procedimiento sugerido por Steel y Torrie (1980), pág 279 y 597:

$$
r_{a(x, y)}=\frac{C O V_{\hat{a}(x, y)}}{\hat{O}_{\hat{a} x} \hat{O}_{\hat{a} y}}
$$

Donde, $C O V_{\hat{a}(x, y)}$ es la covarianza genética entre los caracteres "X" and "Y", mientras que Ôâx $\mathrm{y}$ Ôây son las desviaciones estándar genéticas de los caracteres "X" y "Y" respectivamente.

$$
t=\frac{r_{a(x, y)}}{\sqrt{\frac{1-r_{a(x, y)}^{2}}{n-2}}}
$$

El tamaño efectivo de población se determinó a partir de la función sugerida por Vencovsky (en Resende 2002):

$$
N e=\frac{4 * N_{f} * k_{f}}{K_{f}+3}
$$

Donde, $\mathrm{N}_{\mathrm{f}}=$ Número de familias seleccionadas; mientras que $\mathrm{k}_{\mathrm{f}}$ es el número promedio de individuos seleccionados dentro de familias. Ambos parámetros de la función aumentan con cada nuevo individuo incluido en la selección, en particular si el árbol pertenece a una nueva familia.

\section{RESULTADOS Y DISCUSIÓN}

Como primer resultado se muestra la estimación de los parámetros genéticos poblacionales y sus respectivos valores, con base en la totalidad de los datos previo al raleo silvicultura. Los caracteres cuantitativos (volumen, DAP) registraron valores más altos en los parámetros genéticos, que los cualitativos (calidad, bifurcación), tal y como se observa en el Cuadro 2. Esto se debe a que entre los materiales evaluados (familias y procedencias), se registraron mayores diferencias genéticas en estos caracteres que en los cualitativos (Figura 1 y Cuadros 2 y 7). Los coeficientes

\begin{tabular}{|c|c|c|c|c|c|c|c|}
\hline Parámetros & $\begin{array}{c}\text { Volumen comercial/ } \\
\text { ha }\left(\mathbf{m}^{3}\right)\end{array}$ & Calidad & $\begin{array}{c}\text { DAP año } 1 \\
(\mathbf{c m})\end{array}$ & $\begin{array}{c}\text { DAP año } 4 \\
(\mathrm{~cm})\end{array}$ & $\begin{array}{l}\text { ICA DAP } \\
(\mathbf{c m}) \\
\end{array}$ & Bifurcación & $\begin{array}{c}\text { Altura } \\
\text { bifurcación (m) }\end{array}$ \\
\hline $\mathrm{Va}$ & 417,82 & 0,011 & 1,21 & 6,86 & 0,23 & 0,018 & 0,17 \\
\hline $\mathrm{Ve}$ & 91,45 & 0,029 & 0,56 & 2,82 & 1,53 & 0,15 & 1,15 \\
\hline Vf & 516,23 & 0,041 & 1,78 & 9,81 & 1,77 & 0,17 & 1,35 \\
\hline$h^{2} \mathrm{a}$ & $0,81 \pm 0,17$ & $0,27 \pm 0,10$ & $1,08 \pm 0,20$ & $0,70 \pm 0,16$ & $0,13 \pm 0,07$ & $0,11 \pm 0,06$ & $0,13 \pm 0,07$ \\
\hline $\mathrm{h}^{2} \mathrm{mf}$ & 0,94 & 0,85 & 0,94 & 0,93 & 0,72 & 0,68 & 0,70 \\
\hline Exactitud & 0,97 & 0,92 & 0,97 & 0,96 & 0,85 & 0,82 & 0,84 \\
\hline $\mathrm{h}^{2} \mathrm{ad}$ & 0,77 & 0,21 & 0,61 & 0,64 & 0,10 & 0,083 & 0,10 \\
\hline CVgi\% & 58,64 & 20,35 & 35,57 & 16,60 & 12,33 & 17,81 & 12,76 \\
\hline CVgf $\%$ & 29,32 & 10,17 & 17,78 & 8,30 & 6,16 & 8,91 & 6,38 \\
\hline $\mathrm{CVe} \%$ & 16,71 & 10,20 & 10,65 & 5,15 & 9,23 & 14,97 & 10,01 \\
\hline $\mathrm{CVr}$ & 1,75 & 0,99 & 1,66 & 1,61 & 0,66 & 0,59 & 0,63 \\
\hline SEP & 2,31 & 0,019 & 0,13 & 0,32 & 0,12 & 0,03 & 0,11 \\
\hline Media general & 34,85 & 0,51 & 3,10 & 15,77 & 3,89 & 0,77 & 3,29 \\
\hline
\end{tabular}

Cuadro 2. Parámetros genéticos de veinticinco familias de medios hermanos de A. mangium de cuatro años de edad en Los Chiles, Costa Rica, 2010.

DAP= Diámetro a 1,3 m. ICA DAP = Incremento corriente anual del DIAP.

$\mathrm{Va}=$ varianza genética aditiva. $\mathrm{Ve}=$ varianza residual o no explicada por el modelo. $\mathrm{Vf}=\mathrm{Va}+\mathrm{Vparc}+\mathrm{Ve}$ : varianza fenotípica total. $\mathrm{h}^{2} \mathrm{a}=$ heredabilidad individual en sentido estricto. $\mathrm{h}^{2} \mathrm{mf}=$ heredabilidad media de familia. Exactitud= raíz cuadrada de $\mathrm{h}^{2} \mathrm{mf} . \mathrm{h}^{2} \mathrm{ad}=\mathrm{heredabi}-$ lidad aditiva o dentro de familias. CVgi\%= coeficiente de variación genética aditiva individual. CVgf\%= de variación genotípica entre familias. $\mathrm{CVe} \%=$ coeficiente de variación experimental. $\mathrm{CVr}=$ coeficiente de variación relativa. $\mathrm{SEP}=$ desviación estándar del valor genotípico predicho de progenie. 


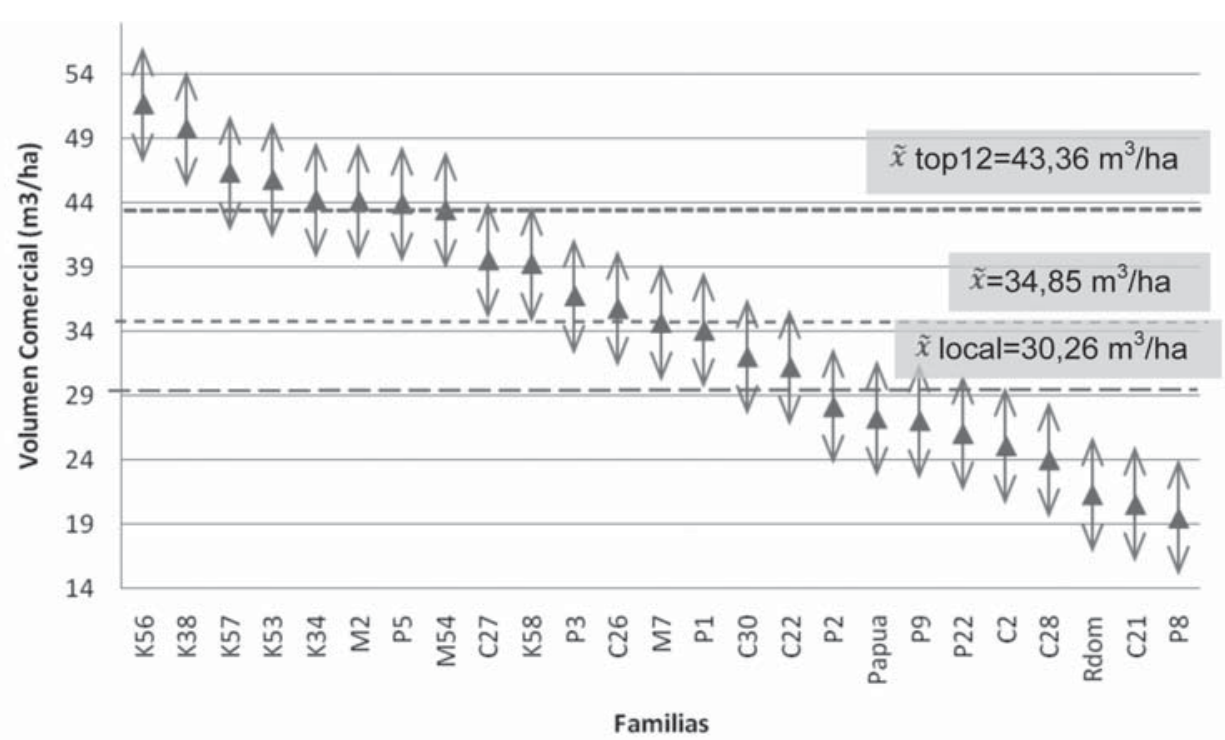

Figura 1. Valores genéticos y límites de confianza para el volumen comercial por hectárea predicho y previo al raleo, en progenies de veinticinco familias de A. mangium de cuatro años de edad. Se indica el promedio de las mejores doce familias, el promedio general de las veinticinco familias y el promedio de las familias locales (C y P). Los Chiles, Costa Rica, 2010.

de variación genéticos aditivos (CVgi) y entre familias (CVgf) estimados en estos caracteres, son un mejor parámetro que puede servir de explicación. Estos dos coeficientes de variación registran valores de más del doble en los caracteres cuantitativos que en los cualitativos, lo que explica una variación mucho mayor en estos caracteres. Los ocho evaluados registraron valores altos de varianza genética aditiva (Va), así como de heredabilidad familiar $\left(\mathrm{h}^{2} \mathrm{mf}\right)$, que variaron entre $68 \%$ y $94 \%$, lo que indica un alto control genético y potencial importante para mejoramiento genético.

A continuación se definen los parámetros presentados en el Cuadro 2, 3, 5 y 8 (Resende 2006):

Va: varianza genética aditiva.

Ve: varianza residual o no explicada por el modelo Vparc: varianza ambiental entre parcelas (familias dentro de bloques).

Vf: Va + Vparc + Ve: varianza fenotípica total.

$\mathrm{h}^{2} \mathrm{a}$ : heredabilidad individual en sentido estricto, es decir, de los efectos aditivos.

$\mathrm{h}^{2} \mathrm{mf}: \frac{0,25 \mathrm{Va}}{0,25 \mathrm{Va}+\frac{\mathrm{Vparc}}{6}+\frac{0,75 \mathrm{Va}+\mathrm{Ve}}{6 * 3}}$ heredabilidad media de familia, asumiendo sobrevivencia completa, donde 6 es el número de repeticiones (o bloques) y 3 el número de individuos/familia/bloque antes del raleo.
Exactitud: raíz cuadrada de $\mathrm{h}^{2} \mathrm{mf}$ : exactitud de selección de progenies, asumiendo sobrevivencia completa.

hªd: $\frac{\mathscr{Q}, 75 \mathrm{Va}}{0,75 \mathrm{Va}+\mathrm{Ve}}$ : heredabilidad aditiva o heredabilidad dentro de familias ("within family").

CVgi\%: [ $(\sqrt[2]{V a}) /$ Media General $] *$ 100: coeficiente de variación genética aditiva individual.

CVgf\%: [ [2 $\sqrt{\frac{V a}{4}}$ /Media General $] * 100$ : coeficiente de variación genotípica entre familias.

CVe\%: $\left\{\sqrt[2]{\frac{0,75^{*} V a+V e}{3}}+\operatorname{Vparc} /\right.$ Media General $\} *$ 100: coeficiente de variación experimental.

CVr: $\mathrm{CVgf} / \mathrm{CVe}=$ coeficiente de variación relativa.

SEP: desviación estándar del valor genotípico predicho de progenie, asumiendo sobrevivencia completa.

\section{Media general del experimento}

La varianza entre parcelas (Vparc) no fue incluida en el Cuadro 2 por presentar valores insignificantes $(<1 \%)$ y por ser de parte de efectos ambientales.

Seguidamente se analiza la base de datos luego de realizar el raleo silvicultural (50\%) dentro de cada subparcela o pareja y se reportan las estimaciones de los parámetros genéticos (Cuadro 3). Se indica el porcentaje de variación de cada parámetro pre y post raleo. 
Con la aplicación de los raleos, se mantiene el mismo patrón de resultados como se espera, pero con valores más bajos en los parámetros genéticos, explicado por la disminución en la cantidad de individuos y por tanto, de la variabilidad del ensayo (Figuras 2 y 3 , Cuadros 3 , 6 y 7). Esta disminución se da especialmente en los valores de heredabilidad de los caracteres cualitativos (altura de bifurcación, presencia de bifurcación y calidad) que se reducen considerablemente, $1150 \%, 40 \%$ y $102 \%$ respectivamente. Esta disminución es esperada debido a que el raleo fue enfocado hacia la eliminación de los árboles de inferior calidad, reduciendo la variabilidad en estos caracteres mucho más que en los caracteres cuantitativos (diámetro y el volumen). Esto sugiere que se debe ejercer precaución al analizar e interpretar los datos de un ensayo genético, dado que resultados bajos en los estimados de heredabilidad no necesariamente significan que un caracter no posea control genético.

Cuadro 3. Parámetros genéticos post raleo (50\%) y porcentaje de variación, de familias de medios hermanos de A. mangium de cuatro años de edad en Los Chiles, Costa Rica, 2010.

\begin{tabular}{|c|c|c|c|c|c|c|c|}
\hline Parámetros & $\begin{array}{c}\text { Volumen comer- } \\
\text { cial/ha }\left(\mathbf{m}^{3}\right)\end{array}$ & Calidad & $\begin{array}{c}\text { DAP } \\
\text { año } 1(\mathrm{~cm}) \\
\end{array}$ & $\begin{array}{c}\text { DAP } \\
\text { año } 4(\mathrm{~cm}) \\
\end{array}$ & $\begin{array}{c}\text { ICA DAP } \\
(\mathrm{cm})\end{array}$ & Bifurcación & $\begin{array}{c}\text { Altura } \\
\text { bifurcación (m) } \\
\end{array}$ \\
\hline Va & $\begin{array}{c}565,78 \\
(\uparrow \% 26,15)\end{array}$ & $\begin{array}{c}0,0024 \\
(\downarrow \% 358,3)\end{array}$ & $\begin{array}{c}1,25 \\
(\uparrow \% 3,2)\end{array}$ & $\begin{array}{c}5,77 \\
(\downarrow \% 18,89)\end{array}$ & $\begin{array}{c}0,25 \\
(\uparrow \% 8)\end{array}$ & $\begin{array}{c}0,012 \\
(\downarrow \% 50)\end{array}$ & $\begin{array}{c}0,0078 \\
(\downarrow \% 2079,4)\end{array}$ \\
\hline Ve & $\begin{array}{c}182,68 \\
(\uparrow \% 49,94)\end{array}$ & $\begin{array}{c}0,032 \\
(\uparrow \% 9,37)\end{array}$ & $\begin{array}{c}0,64 \\
(\uparrow \% 12,5)\end{array}$ & $\begin{array}{c}2,99 \\
(\uparrow \% 5,68)\end{array}$ & $\begin{array}{c}1,74 \\
(\uparrow \% 12,06) \\
\end{array}$ & $\begin{array}{c}0,17 \\
(\uparrow \% 11,76)\end{array}$ & $\begin{array}{c}1,21 \\
(\uparrow \% 4,95)\end{array}$ \\
\hline Vf & $\begin{array}{c}762,14 \\
(\uparrow \% 32,27)\end{array}$ & $\begin{array}{c}0,035 \\
(\downarrow \% 17,14)\end{array}$ & $\begin{array}{c}1,91 \\
(\uparrow \% 6,80)\end{array}$ & $\begin{array}{c}8,81 \\
(\downarrow \% 11,35)\end{array}$ & $\begin{array}{c}2,01 \\
(\uparrow \% 11,94)\end{array}$ & $\begin{array}{c}0,18 \\
(\uparrow \% 5,55)\end{array}$ & $\begin{array}{c}1,26 \\
(\downarrow \% 7,14)\end{array}$ \\
\hline$h^{2} a$ & $\begin{array}{c}0,74 \pm 0,21 \\
(\downarrow \% 8,46) \\
\end{array}$ & $\begin{array}{c}0,07 \pm 0,06 \\
(\downarrow \% 75,3) \\
\end{array}$ & $\begin{array}{c}0,65 \pm 0,19 \\
(\downarrow \% 3,48) \\
\end{array}$ & $\begin{array}{c}0,65 \pm 0,19 \\
(\downarrow \% 6,23) \\
\end{array}$ & $\begin{array}{c}0,12 \pm 0,08 \\
(\downarrow \% 3,8) \\
\end{array}$ & $\begin{array}{c}0,06 \pm 0,06 \\
(\downarrow \% 38,84) \\
\end{array}$ & $\begin{array}{c}0,006 \pm 0,02 \\
(\downarrow \% 95,26)\end{array}$ \\
\hline $\mathrm{h}^{2} \mathrm{mf}$ & $\begin{array}{c}0,90 \\
(\downarrow \% 4,44)\end{array}$ & $\begin{array}{c}0,42 \\
(\downarrow \% 102,3) \\
\end{array}$ & $\begin{array}{c}0,90 \\
(\downarrow \% 4,44) \\
\end{array}$ & $\begin{array}{c}0,90 \\
(\downarrow \% 3,33) \\
\end{array}$ & $\begin{array}{c}0,59 \\
(\downarrow \% 22,03) \\
\end{array}$ & $\begin{array}{c}0,40 \\
(\downarrow \% 67,5) \\
\end{array}$ & $\begin{array}{c}0,056 \\
(\downarrow \% 1150) \\
\end{array}$ \\
\hline Exactitud & $\begin{array}{c}0,95 \\
(\downarrow \% 2,11) \\
\end{array}$ & $\begin{array}{c}0,65 \\
(\downarrow \% 41,53) \\
\end{array}$ & $\begin{array}{c}0,94 \\
(\downarrow \% 3,19) \\
\end{array}$ & $\begin{array}{c}0,94 \\
(\downarrow \% 2,12) \\
\end{array}$ & $\begin{array}{c}0,77 \\
(\downarrow \% 10,38) \\
\end{array}$ & $\begin{array}{c}0,63 \\
(\downarrow \% 30,15) \\
\end{array}$ & $\begin{array}{c}0,23 \\
(\downarrow \% 265,2) \\
\end{array}$ \\
\hline$h^{2} \mathrm{ad}$ & $\begin{array}{c}0,69 \\
(\downarrow \% 11,59) \\
\end{array}$ & $\begin{array}{c}0,051 \\
(\downarrow \% 311,7) \\
\end{array}$ & $\begin{array}{c}0,59 \\
(\downarrow \% 3,38) \\
\end{array}$ & $\begin{array}{c}0,59 \\
(\downarrow \% 8,47) \\
\end{array}$ & $\begin{array}{c}0,09 \\
(\downarrow \% 11,11) \\
\end{array}$ & $\begin{array}{c}0,05 \\
(\downarrow \% 66)\end{array}$ & $\begin{array}{c}0,004 \\
(\downarrow \% 2400)\end{array}$ \\
\hline CVgi\% & $\begin{array}{c}49,94 \\
(\downarrow \% 17,42) \\
\end{array}$ & $\begin{array}{c}8,98 \\
(\downarrow \% 126,6) \\
\end{array}$ & $\begin{array}{c}36,33 \\
(\uparrow \% 2,09) \\
\end{array}$ & $\begin{array}{c}14,89 \\
(\downarrow \% 11,48) \\
\end{array}$ & $\begin{array}{c}12,73 \\
(\uparrow \% 3,14) \\
\end{array}$ & $\begin{array}{c}14,81 \\
(\downarrow \% 20,25) \\
\end{array}$ & $\begin{array}{c}2,57 \\
(\downarrow \% 396,4) \\
\end{array}$ \\
\hline $\mathrm{CVgf} \%$ & $\begin{array}{c}24,97 \\
(\downarrow \% 17,42)\end{array}$ & $\begin{array}{c}4,49 \\
(\downarrow \% 126,5) \\
\end{array}$ & $\begin{array}{c}18,16 \\
(\uparrow \% 2,09)\end{array}$ & $\begin{array}{c}7,44 \\
(\downarrow \% 11,55)\end{array}$ & $\begin{array}{c}6,36 \\
(\uparrow \% 3,14) \\
\end{array}$ & $\begin{array}{c}7,41 \\
(\downarrow \% 20,24)\end{array}$ & $\begin{array}{c}1,28 \\
(\downarrow \% 398,4)\end{array}$ \\
\hline $\mathrm{CVe} \%$ & $\begin{array}{c}19,86 \\
(\uparrow \% 15,86)\end{array}$ & $\begin{array}{c}12,68 \\
(\uparrow \% 19,55)\end{array}$ & $\begin{array}{c}14,76 \\
(\uparrow \% 27,84)\end{array}$ & $\begin{array}{c}6,06 \\
(\uparrow \% 15,01)\end{array}$ & $\begin{array}{c}12,80 \\
(\uparrow \% 27,89)\end{array}$ & $\begin{array}{c}21,95 \\
(\uparrow \% 31,79)\end{array}$ & $\begin{array}{c}12,91 \\
(\uparrow \% 22,46)\end{array}$ \\
\hline $\mathrm{CVr}$ & $\begin{array}{c}1,25 \\
(\downarrow \% 40) \\
\end{array}$ & $\begin{array}{c}0,35 \\
(\downarrow \% 182,8) \\
\end{array}$ & $\begin{array}{c}1,23 \\
(\downarrow \% 34,95) \\
\end{array}$ & $\begin{array}{c}1,22 \\
(\downarrow \% 31,96)\end{array}$ & $\begin{array}{c}0,49 \\
(\downarrow \% 34,69) \\
\end{array}$ & $\begin{array}{c}0,33 \\
(\downarrow \% 78,78) \\
\end{array}$ & $\begin{array}{c}0,099 \\
(\downarrow \% 536,3)\end{array}$ \\
\hline SEP & $\begin{array}{c}3,67 \\
(\uparrow \% 37,06) \\
\end{array}$ & $\begin{array}{c}0,018 \\
(\downarrow \% 5,55) \\
\end{array}$ & $\begin{array}{c}0,17 \\
(\uparrow \% 23,52) \\
\end{array}$ & $\begin{array}{c}0,37 \\
(\uparrow \% 13,51) \\
\end{array}$ & $\begin{array}{c}0,15 \\
(\uparrow \% 20) \\
\end{array}$ & $\begin{array}{c}0,042 \\
(\uparrow \% 28,57) \\
\end{array}$ & $\begin{array}{c}0,042 \\
(\downarrow \% 161,9) \\
\end{array}$ \\
\hline Media general & $\begin{array}{c}47,62 \\
(\uparrow \% 26,82) \\
\end{array}$ & $\begin{array}{c}0,54 \\
(\uparrow \% 5,55) \\
\end{array}$ & $\begin{array}{c}3,08 \\
(\downarrow \% 0,64)\end{array}$ & $\begin{array}{c}16,14 \\
(\uparrow \% 2,29)\end{array}$ & $\begin{array}{c}3,93 \\
(\uparrow \% 1,01) \\
\end{array}$ & $\begin{array}{c}0,74 \\
(\downarrow \% 4,05)\end{array}$ & $\begin{array}{c}3,42 \\
(\uparrow \% 3,80) \\
\end{array}$ \\
\hline
\end{tabular}

DAP= Diámetro a 1,3 m. ICA DAP = Incremento corriente anual del DIAP.

$\mathrm{Va}=$ varianza genética aditiva. $\mathrm{Ve}=$ varianza residual o no explicada por el modelo. $\mathrm{Vf}=\mathrm{Va}+\mathrm{Vparc}+\mathrm{Ve}$ : varianza fenotípica total. $\mathrm{h}^{2} \mathrm{a}=$ heredabilidad individual en sentido estricto. $\mathrm{h}^{2} \mathrm{mf}=$ heredabilidad media de familia. Exactitud= raíz cuadrada de $\mathrm{h}^{2} \mathrm{mf} . \mathrm{h}^{2} \mathrm{ad}=\mathrm{heredabi-}$ lidad aditiva o dentro de familias. CVgi\%= coeficiente de variación genética aditiva individual. CVgf\%= de variación genotípica entre familias. $\mathrm{CVe} \%=$ coeficiente de variación experimental. $\mathrm{CVr}=$ coeficiente de variación relativa. $\mathrm{SEP}=$ desviación estándar del valor genotípico predicho de progenie. 
Los coeficientes de variación genética disminuyen pero los del error ( $\mathrm{CVe} \%)$ se incrementaron levemente, lo que reduce los Coeficientes de Variación relativa en todos los caracteres investigados. Las medias generales tienden a incrementarse levemente luego del raleo. La única excepción es el DAP al año 1, pero la diferencia fue casi nula. En el caracter "presencia de bifurcación", la disminución del valor refleja una mejoría en la característica, ya que 1 equivale a bifurcación presente y 0 a ausente.

Las mismas familias se ubican en los primeros diez puestos del ranking del volumen comercial por hectárea, así también, las peores diez familias se ubican en las posiciones más bajas después del raleo (Cuadro 6 y Figura 3). Por tanto, la eliminación del individuo de menor crecimiento o calidad de cada pareja inicial, no afectó la estructura genética del ensayo. Puede inclusive argumentarse que este diseño es una variante en el tiempo del diseño de árboles individuales (Single Tree Plot), ya que después del raleo queda en terreno precisamente este diseño genético. Pero incorpora mejoras, porque logra reducir el desbalance inicial causado por la mortalidad o errores de establecimiento, más la eliminación de los peores representantes de cada familia. De modo que el análisis genético ocurre finalmente con los mejores representantes de cada familia y con un menor nivel de desbalance, en este caso la mortalidad se redujo de un $29 \%$ a un $15 \%$ (Cuadro 5). Una vez efectuado el raleo silvicultural de un $50 \%$ de los árboles, se disminuye la competencia y puede continuar por algunos años más generando información genética valiosa. Finalmente, posterior al raleo el ensayo se convierte automáticamente en un huerto semillero.

En el Cuadro 4 se presentan los resultados de las correlaciones genéticas entre los caracteres investigados de acuerdo con la base de datos post raleo.

El volumen comercial por hectárea se correlaciona en gran medida con el ICA del DAP, pero no así con las variables que describen la calidad del árbol (calidad, bifurcación, altura de bifurcación). El volumen comercial y los tres caracteres de calidad registran una correlación prácticamente nula entre sí. La variable calidad está determinada en gran medida por ambas variables de bifurcación, por tanto, a mayor altura de bifurcación mejor es la calidad del árbol $(0,74)$ y a mayor presencia de bifurcación peor es la calidad $(-0,37)$.

Los caracteres cuantitativos estuvieron positivamente correlacionados entre sí. El diámetro al año 1 estuvo fuertemente relacionado con el del año $4(\mathrm{r}=0,83)$. Por lo tanto, el potencial de crecimiento diamétrico que exhibieron los árboles se expresó desde temprana edad y se ha mantenido de manera robusta $(r=0,83)$ hasta el año 4. Esto podría ser utilizado operativamente, dado que permitiría realizar selección temprana en esta especie. Esta correlación juvenil-adulto tan alta para el DAP, puede ser explicada en relación con la tasa de crecimiento tan acelerada de esta especie, y a que, la A. mangium es una de un ciclo de vida corto (se estima menor a 15-20 años CATIE 1992).

Cuadro 4. Matriz de correlaciones genéticas post-raleo de los caracteres medidos en familias de medios hermanos de A. mangium de cuatro años de edad en Los Chiles, Costa Rica, 2010. ${ }^{1}$

\begin{tabular}{|c|c|c|c|c|c|c|c|}
\hline Caracter & Calidad & $\begin{array}{c}\text { DAP } \\
\text { año } 1(\mathrm{~cm})\end{array}$ & $\begin{array}{c}\text { DAP } \\
\text { año } 4(\mathrm{~cm}) \\
\end{array}$ & $\begin{array}{c}\text { ICA DAP } \\
(\mathrm{cm})\end{array}$ & Bifurcación & $\begin{array}{c}\text { Altura } \\
\text { bifurcación (m) }\end{array}$ & $\begin{array}{c}\text { Volumen comer- } \\
\text { cial/ha }\left(\mathbf{m}^{3}\right)\end{array}$ \\
\hline Calidad & 1,00 & $-0,22 * *$ & $-0,36 * *$ & $-0,35 * *$ & $-0,37 * *$ & $0,74 * * *$ & $-0,04 \mathrm{~ns}$ \\
\hline DAP año 1 & $-0,22 * *$ & 1,00 & $0,83 * * *$ & $0,75 * * *$ & $-0,18 * *$ & $0,13 * *$ & $0,73 * * *$ \\
\hline DAP año 4 & $-0,36 * *$ & $0,83 * * *$ & 1,00 & $0,79 * * *$ & $0,06 \mathrm{~ns}$ & $-0,09 \mathrm{Ns}$ & $0,88 * * *$ \\
\hline ICA DAP & $-0,35^{* *}$ & $0,75^{* * *}$ & $0,79 * * *$ & 1,00 & $0,08 \mathrm{~ns}$ & $-0,17 * *$ & $0,61 * * *$ \\
\hline Presencia de bifurcación & $-0,37 * *$ & $-0,18 * *$ & $0,06 \mathrm{~ns}$ & $0,08 \mathrm{~ns}$ & 1,00 & $-0,79 * * *$ & $0,00 \mathrm{~ns}$ \\
\hline Altura bifurcación & $0,74 * * *$ & $0,13 * *$ & $-0,09 \mathrm{~ns}$ & $-0,17 * *$ & $-0,79 * * *$ & 1,00 & $0,15 * *$ \\
\hline Volumen comercial/ha & $-0,04 \mathrm{~ns}$ & $0,73 * * *$ & $0,88 * * *$ & $0,61 * * *$ & $0,00 \mathrm{~ns}$ & $0,15 * *$ & 1,00 \\
\hline
\end{tabular}

DAP= Diámetro a 1,3 m. ICA DAP = Incremento corriente anual del DIAP.

${ }^{1}$ Significancia de la correlación: $\mathrm{ns}=$ no significativo; $*=\mathrm{p}<0,05 ; * * \mathrm{p}<0,01 ; * * *=\mathrm{p}<0,001$. 
Cuadro 5. Parámetros genéticos relativos a la supervivencia en un ensayo de progenies de A. mangium de cuatro años de edad en Los Chiles, Costa Rica, 2010.

\begin{tabular}{lcc}
\hline $\begin{array}{l}\text { Parámetros } \\
\text { genéticos }\end{array}$ & $\begin{array}{c}\text { Supervivencia pre } \\
\text { raleo }\end{array}$ & $\begin{array}{c}\text { Supervivencia } \\
\text { post raleo }\end{array}$ \\
\hline Va & 0,019 & 0,015 \\
$\mathrm{Ve}$ & 0,179 & 0,109 \\
$\mathrm{Vf}$ & 0,205 & 0,126 \\
$\mathrm{~h}^{2} \mathrm{a}$ & $0,09 \pm 0,04$ & $0,12 \pm 0,08$ \\
$\mathrm{~h}^{2} \mathrm{mf}$ & 0,615 & 0,607 \\
Exactitud & 0,784 & 0,779 \\
$\mathrm{~h}^{2}$ ad & 0,073 & 0,094 \\
$\mathrm{CVgi} \%$ & 19,53 & 14,43 \\
$\mathrm{CVgf} \%$ & 9,76 & 7,22 \\
$\mathrm{CVe} \%$ & 18,90 & 14,22 \\
$\mathrm{CVr}$ & 0,51 & 0,51 \\
$\mathrm{SEP}$ & 0,043 & 0,039 \\
Media general $(\%)$ & 70,67 & 85,0 \\
\hline
\end{tabular}

$\mathrm{Va}=$ varianza genética aditiva. $\mathrm{Ve}=$ varianza residual o no explicada por el modelo. $\mathrm{Vf}=\mathrm{Va}+\mathrm{Vparc}+\mathrm{Ve}$ : varianza fenotípica total. $\mathrm{h}^{2} \mathrm{a}=$ heredabilidad individual en sentido estricto. $\mathrm{h}^{2} \mathrm{mf}=$ heredabilidad media de familia. Exactitud= raíz cuadrada de $\mathrm{h}^{2} \mathrm{mf} . \mathrm{h}^{2} \mathrm{ad}=$ heredabilidad aditiva o dentro de familias. $\mathrm{CVgi} \%=$ coeficiente de variación genética aditiva individual. $\mathrm{CVgf} \%=\mathrm{de}$ variación genotípica entre familias. $\mathrm{CVe} \%=$ coeficiente de variación experimental. $\mathrm{CVr}=$ coeficiente de variación relativa. $\mathrm{SEP}=$ desviación estándar del valor genotípico predicho de progenie.

La calidad del árbol se correlaciona inversamente proporcional con el incremento en diámetro $(r=-0,35)$. $\mathrm{Al}$ año 1, el DAP exhibió una correlación negativa de $r=-0,22$ con la calidad, que se acentuó al año cuatro donde aumentó a $r=-0,36$. Estos datos sugieren que a mayor tasa de incremento en diámetro, la calidad del fuste muestra una tendencia a disminuir. Sin embargo, al no registrarse una correlación entre el volumen comercial/ha y la calidad $(r=-0,04)$, no hay evidencia de que exista una relación genética inversa entre estos caracteres cuantitativos y la calidad del árbol.

La variable supervivencia mostró un alto control genético (Cuadro 5), lo que indica que algunas familias se adaptan mejor al sitio $\left(\mathrm{h}^{2} \mathrm{mf}=0,615\right.$ y 0,607$)$. La media general indica que un $70,67 \%$ de los individuos del ensayo se encontraban vivos al momento de la medición a los cuatro años. Los resultados post raleo
Cuadro 6. Ranking, efectos genéticos aditivos, ganancia y nuevo promedio para la variable volumen comercial por hectárea para las veinticinco familias de progenies de A. mangium de cuatro años de edad, en Los Chiles, Costa Rica, $2010 .{ }^{1}$

\begin{tabular}{|c|c|c|c|}
\hline Pos. & Familia & $\begin{array}{c}\text { Ganancia gené- } \\
\text { tica }\left(\mathbf{m}^{3} / \mathrm{ha}\right)\end{array}$ & $\begin{array}{l}\text { Nueva } \\
\text { media }\end{array}$ \\
\hline 1 & K57 (+3) & 29,91 & 77,54 \\
\hline 2 & P5 (+5) & 28,36 & 75,99 \\
\hline 3 & M2 (+3) & 27,59 & 75,22 \\
\hline 4 & K53 (=) & 27,16 & 74,79 \\
\hline 5 & K38 (-3) & 26,52 & 74,15 \\
\hline 6 & $\mathrm{C} 27(+\mathbf{3})$ & 25,84 & 73,47 \\
\hline 7 & M54 (+1) & 25,19 & 72,82 \\
\hline 8 & K34 (-3) & 24,61 & 72,24 \\
\hline 9 & K56 (-8) & 24,16 & 71,79 \\
\hline 10 & K58 (=) & 23,10 & 70,73 \\
\hline 11 & $\mathrm{C} 26(+\mathbf{1})$ & 21,40 & 69,02 \\
\hline 12 & P3 (-1) & 19,53 & 67,16 \\
\hline 13 & M7 (=) & 17,89 & 65,52 \\
\hline 14 & C30 (+1) & 16,40 & 64,03 \\
\hline 15 & P1 (-1) & 14,88 & 62,51 \\
\hline 16 & C22 (=) & 13,41 & 61,04 \\
\hline 17 & P22 (+3) & 11,67 & 59,30 \\
\hline 18 & Papua (=) & 10,04 & 57,67 \\
\hline 19 & C28 (+3) & 8,50 & 56,13 \\
\hline 20 & C2 (+1) & 7,09 & 54,72 \\
\hline 21 & P2 (-3) & 5,70 & 53,33 \\
\hline 22 & P9 (-3) & 4,36 & 51,99 \\
\hline 23 & C21 (+1) & 2,91 & 50,54 \\
\hline 24 & Rdom (-1) & 1,53 & 49,16 \\
\hline 25 & P8 (=) & 0,00 & 47,62 \\
\hline
\end{tabular}

${ }^{1}$ El número entre paréntesis indica el cambio de posición dentro del ranking luego de realizado el raleo.

reducen la mortalidad en un $15 \%$ (en este caso la mortalidad se da solo cuando ambos árboles de cada pareja estaban muertos). De toda la varianza fenotípica registrada post raleo del caracter sobrevivencia, sólo un $11,9 \%$ corresponde a la varianza genética aditiva. Si se utilizaran las diez mejores familias en cuanto a adaptabilidad al sitio (C27, P3, K53, K56, C26, K34, 


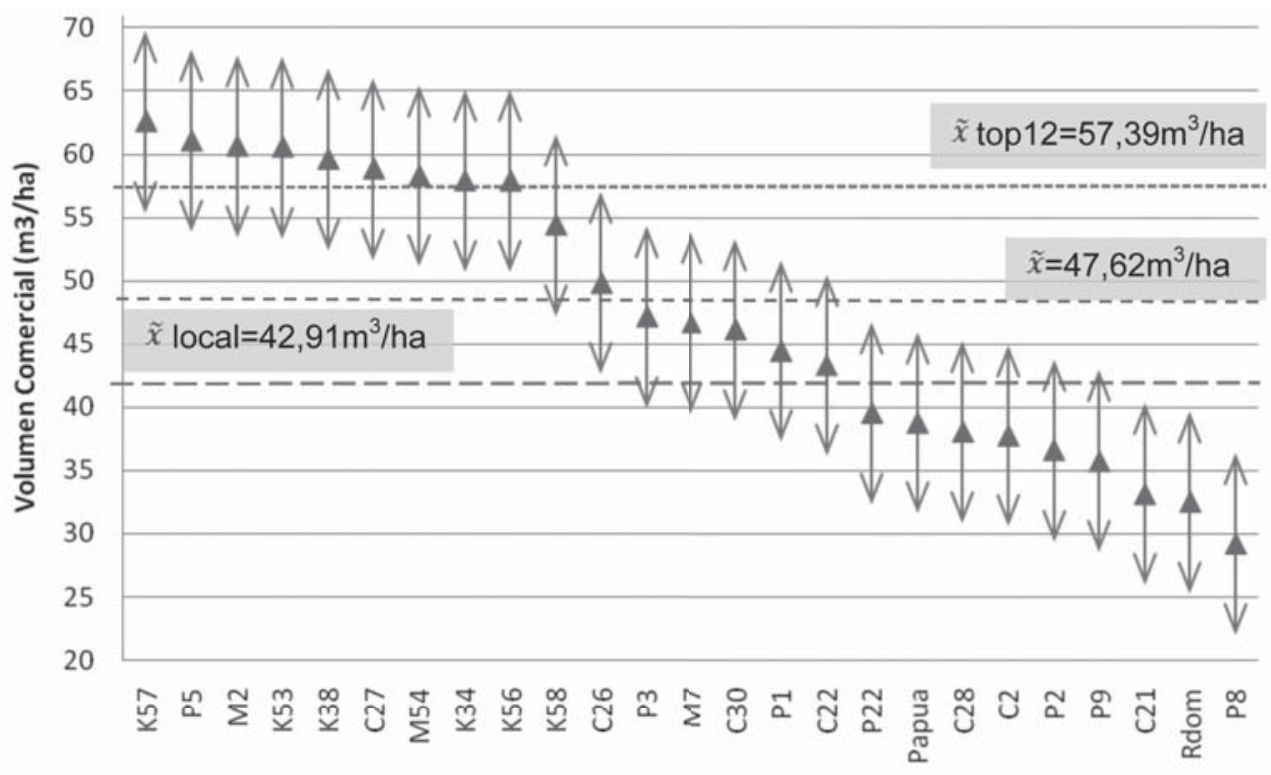

Familias

Figura 2. Valores genéticos y límites de confianza para el volumen comercial por hectárea predicho posterior al raleo, en un ensayo de progenies de A. mangium de cuatro años de edad. Se indica el promedio de las mejores doce familias, el promedio general de las veinticinco familias y el promedio de las familias locales (C y P). en Los Chiles, Costa Rica, 2010.

K38, K57, P1, P5), la supervivencia de los árboles aumentaría al $92,76 \%$ según los resultados post raleo. Las mejores familias son las procedentes de Kanguroid (Colombia) y Parrita. Llama la atención que las familias de la procedencia El Concho (la más cercana al sitio del ensayo) registren los peores valores de adaptabilidad. Posiblemente tenga relación con que las familias del Concho, también registraron colectivamente los peores crecimientos. El raleo a los cuatro años de edad se puede considerar como tardío para una plantación de A. mangium establecida con 1111 árboles/ha iniciales (CATIE 1992), y pudo provocar una mortalidad relativamente mayor en aquellas familias de menor vigor y crecimiento inicial, circundadas por materiales con mayor tasa de crecimiento. Este fenómeno es conocido como competencia intergenotípica y puede afectar sensiblemente el comportamiento de los materiales, si todos sus vecinos que lo rodeen lo superan en vigor (Resende 2002). La mortalidad puede registrar efectos confundidos y en ocasiones, es difícil separar si la tasa de sobrevivencia se debe a pobre adaptabilidad al sitio, o a errores en el establecimiento y manejo del ensayo (Badilla et al. 2002).

En los resultados de los Cuadros 2, 3 y 5, los valores de exactitud de selección de progenies se mantienen altos $(>0,63)$ excepto en el caracter altura de bifurcación (Cuadro 3).

El cálculo de la variable Volumen Comercial por hectárea se realiza integrando todas las variables de calidad, crecimiento y mortalidad. Es, por lo tanto, un indicador del valor económico real del árbol que puede ser analizado genéticamente y permite determinar el mejor material genético de acuerdo a los objetivos de la plantación: obtener la mayor cantidad de madera de la mejor calidad posible con turnos de corta reducidos y con la menor inversión en mantenimientos y replantes (Figura 1).

Entre la mejor familia (K56) y la peor (P8) hay una diferencia de $32,15 \mathrm{~m}^{3} /$ ha $(265 \%)$ (Figura 1). Las mejores dos familias exhiben ya diferencias significativas, desde la novena familia (C27) hacia abajo en el ranking genético. Las primeras ocho 


\begin{tabular}{|c|c|c|}
\hline Orden & Sin raleo & Con Raleo \\
\hline 1 & K56 & K57 \\
\hline 2 & K38 & P5 \\
\hline 3 & K57 & M2 \\
\hline 4 & K53 & K53 \\
\hline 5 & K34 & K38 \\
\hline 6 & M2 & C27 \\
\hline 7 & P5 & M54 \\
\hline 8 & M54 & K34 \\
\hline 9 & $\mathrm{C} 27$ & K56 \\
\hline 10 & K58 & - K58 \\
\hline 11 & P3 & - $c 26$ \\
\hline 12 & $\mathrm{C} 26$ & P3 \\
\hline 13 & M7 & M7 \\
\hline 14 & P1 & C30 \\
\hline 15 & С $30-$ & P1 \\
\hline 16 & $\mathrm{C} 22-$ & $-c 22$ \\
\hline 17 & P2 & P22 \\
\hline 18 & Papua & Papua \\
\hline 19 & P9 & C28 \\
\hline 20 & P22 & $\mathrm{C} 2$ \\
\hline 21 & $\mathrm{C} 2$ & P2 \\
\hline 22 & C28 & P9 \\
\hline 23 & Rdom . & $\mathrm{C} 21$ \\
\hline 24 & $\mathrm{C} 21-$ & Rdom \\
\hline 25 & P8 & P8 \\
\hline
\end{tabular}

Figura 3. Efecto del raleo en el movimiento de familias de $A$. mangium en el ranking genético a los cuatro años de edad en el volumen comercial por hectárea. Los Chiles, Costa Rica, 2010.

familias no presentan diferencias significativas entre ellas.

El promedio de las doce mejores familias ( $x=43,36$ $\mathrm{m}^{3} / \mathrm{ha}$ ) supera en $8,51 \mathrm{~m}^{3} /$ ha al promedio general con veinticinco familias evaluadas. El producto de este diferencial de selección con la heredabilidad familiar $(0,94)$, estima una ganancia genética en este programa de un $22,9 \%$ para el volumen comercial por hectárea.

Luego del raleo (Figura 2), las primeras once familias no presentan diferencias significativas y se mantiene una tendencia similar en el ranking 3 (Figura 3 y Cuadro 6). El promedio de las mejores doce familias es ahora de $57,39 \mathrm{~m}^{3} / \mathrm{ha}$, el diferencial de selección correspondiente es de $9,77 \mathrm{~m}^{3} /$ ha y la heredabilidad media familiar $\left(h^{2} \mathrm{mf}\right)$ es ahora de 0,90 Por tanto, la ganancia genética respectiva se estima en un 18,5\%.
El raleo de las subparcelas modifica levemente las posiciones de las familias en el ranking del volumen comercial por hectárea, pero la tendencia general de superioridad de las familias con código $\mathrm{K}$ y M se mantiene. Se observan tres grupos definidos dentro del ranking: posiciones 1-9, 10-16 y 17-25. Puede también observarse, que tanto las mejores 10 familias, como las peores diez familias en el ranking del volumen comercial/ha se mantienen después del raleo.

Una plantación establecida con semilla mejorada de un huerto semillero con estos veinticinco árboles, tendría $91,65 \mathrm{~m}^{3} /$ ha de madera comercial a los cuatro años de edad (Cuadro 7). Este valor supera en 44,03 $\mathrm{m}^{3}(92,5 \%)$ al promedio individual poblacional.

Se utiliza la restricción de dos individuos por familia para que el tamaño efectivo poblacional (y por tanto la base genética del ensayo) se mantenga alta. Se estima finalmente una ganancia genética del 40,8\% en la variable Volumen Comercial por hectárea, seleccionando las doce mejores familias y luego los dos mejores árboles de cada una de estas. Una plantación establecida con este material alcanzaría a los cuatro años de edad $(\mathrm{N}=1111)$ en Los Chiles, zona norte, $91,65 \mathrm{~m}^{3} /$ ha con un mínimo $10 \mathrm{~cm}$ de diámetro y sin madera defectuosa $\left(I M A=22,9 \mathrm{~m}^{3} / \mathrm{ha} / \mathrm{año}\right)$.

$\mathrm{GG}=\mathrm{S} \times \mathrm{h}^{2} \mathrm{mf}+\mathrm{S} \times \mathrm{h}^{2} \mathrm{ad}$

$\mathrm{GG}=8,79 \mathrm{~m}^{3} / \mathrm{ha}+\left(34,84 \mathrm{~m}^{3} / \mathrm{ha}\right) \times 0,69$

$\mathrm{GG}=8,79 \mathrm{~m}^{3} / \mathrm{ha}+24,04 \mathrm{~m}^{3} / \mathrm{ha}=32,83 \mathrm{~m}^{3} / \mathrm{ha}=40,8 \%$

Importante también es analizar los materiales desde el punto de vista de su origen geográfico, información vital para intentar ampliar la base genética a futuro. Se presenta entonces en el Cuadro 8, un análisis por procedencia, sin distinción de la estructura familiar.

A nivel de procedencias, familias y árboles individuales, las accesiones con código Kanguroid y Cordoba (procedencias derivadas de Colombia) son las mejor posicionadas en el ranking genético (Figuras 1 y 2, Cuadros 6 y 7). Mientras que individualmente las familias Parrita 5, Concho 27 y Concho 26, también presentan buenos resultados y se logran filtrar en las primeras posiciones, lo que incrementaría la diversidad genética en un futuro huerto semillero junto con los materiales colombianos.

Puede observarse del Cuadro 8 una tendencia similar a la del análisis de progenies por familias mostrado anteriormente. Las heredabilidades se mantienen muy elevadas en todos los caracteres 
Cuadro 7. Parámetros genéticos de los mejores dos individuos de las mejores doce familias post raleo de A. mangium a los cuatro años de edad en su volumen comercial por hectárea. Los Chiles, Costa Rica, 2010.

\begin{tabular}{lcccccc}
\hline Posición & Familia & f & a & Ganancia & $\begin{array}{c}\text { Nueva Me- } \\
\text { dia }\end{array}$ & Ne \\
\hline 1 & K57 & 145,08 & 72,02 & 72,02 & 119,64 & 1,00 \\
2 & M2 & 131,34 & 63,06 & 67,54 & 115,16 & 2,00 \\
3 & K53 & 130,39 & 61,91 & 65,66 & 113,28 & 3,00 \\
4 & K38 & 126,57 & 57,96 & 63,74 & 111,36 & 4,00 \\
5 & M2 & 123,98 & 57,92 & 62,57 & 110,19 & 4,71 \\
6 & P5 & 119,17 & 52,93 & 60,97 & 108,59 & 5,71 \\
7 & M54 & 114,89 & 50,28 & 59,44 & 107,06 & 6,72 \\
8 & K38 & 115,80 & 47,91 & 58,00 & 105,62 & 7,38 \\
9 & M54 & 111,21 & 47,90 & 56,88 & 104,50 & 8,00 \\
10 & K57 & 112,65 & 47,90 & 55,98 & 103,60 & 8,57 \\
11 & K56 & 109,70 & 47,64 & 55,22 & 102,84 & 9,63 \\
12 & K56 & 112,56 & 45,26 & 54,39 & 102,01 & 10,18 \\
13 & K53 & 109,59 & 44,07 & 53,60 & 101,22 & 10,71 \\
14 & P5 & 99,85 & 42,54 & 52,81 & 100,43 & 11,20 \\
15 & K34 & 103,88 & 42,11 & 52,09 & 99,71 & 12,31 \\
16 & C27 & 96,44 & 35,97 & 51,08 & 98,70 & 13,40 \\
17 & C27 & 95,44 & 35,27 & 50,15 & 97,77 & 13,91 \\
18 & K34 & 88,71 & 32,08 & 49,15 & 96,77 & 14,40 \\
19 & C26 & 90,94 & 30,27 & 48,16 & 95,78 & 15,51 \\
20 & C26 & 93,97 & 30,24 & 47,26 & 94,88 & 16,00 \\
21 & P3 & 87,69 & 29,19 & 46,40 & 94,02 & 17,11 \\
22 & P3 & 84,48 & 27,87 & 45,56 & 93,18 & 17,60 \\
23 & K58 & 81,63 & 27,42 & 44,77 & 92,39 & 18,71 \\
24 & K58 & 82,38 & 27,14 & 44,03 & 91,65 & 19,20 \\
\hline & & & & & & \\
\hline
\end{tabular}

f: valor fenotípico individual;

a: efecto genético aditivo predicho;

Nueva media si se plantara solamente la semilla de este individuo y de todos los demás que lo superen en el ranking.

Ne: tamaño efectivo poblacional;

cuantitativos y en el carácter calidad, excepto para los relacionados con la bifurcación (presencia de bifurcación y altura de bifurcación).

En términos de ranking genético para el volumen comercial/ha, claramente las procedencias derivadas Kanguroid y Córdoba (ambas de Córdoba, Colombia) no presentan diferencias significativas entre ellas, pero si con relación a las otras cuatro procedencias. Mientras que las de República Dominicana y Papúa
Nueva Guinea (esta última la única procedencia autóctona), se ubicaron en los últimos lugares del ranking genético (Figura 4).

Solamente las dos mejores procedencias (origen colombiano) estuvieron por encima del promedio global del ensayo (45,65 $\mathrm{m}^{3} / \mathrm{ha}$ ) (Cuadro 9). Si se seleccionan únicamente estas dos derivadas como fuentes semilleras, el nuevo promedio de toda su progenie produciría un volumen de $58,19 \mathrm{~m}^{3} /$ ha, que representa una ganancia 
Cuadro 8. Estimaciones de parámetros genéticos post raleo en un ensayo de seis procedencias de A. mangium a los cuatro años de edad, en Los Chiles, Costa Rica, 2010.

\begin{tabular}{lcccccccc}
\hline Parámetros & $\begin{array}{c}\text { Volumen co- } \\
\text { mercial/ha }\end{array}$ & $\begin{array}{c}\text { Volumen } \\
\text { comercial }\end{array}$ & Calidad & $\begin{array}{c}\text { DAP } \\
\text { año 1 }\end{array}$ & $\begin{array}{c}\text { DAP } \\
\text { año 4 }\end{array}$ & $\begin{array}{c}\text { ICA DAP } \\
\text { Presencia de } \\
\text { bifurcación }\end{array}$ & $\begin{array}{c}\text { Altura de tro- } \\
\text { za bifurcada }\end{array}$ \\
\hline $\mathrm{Vg}$ & 126,84 & 0,0001 & 0,0005 & 0,39 & 1,27 & 0,14 & 0,0001 & 0,001 \\
$\mathrm{Ve}$ & 661,14 & 0,0007 & 0,03 & 1,64 & 7,61 & 1,90 & 0,18 & 1,24 \\
$\mathrm{Vf}$ & 807,75 & 0,0008 & 0,04 & 2,06 & 8,90 & 2,10 & 0,19 & 1,28 \\
h $^{2} \mathrm{mf}$ & 0,95 & 0,90 & 0,57 & 0,97 & 0,96 & 0,86 & 0,08 & 0,06 \\
Exactitud & 0,97 & 0,95 & 0,75 & 0,98 & 0,98 & 0,93 & 0,27 & 0,25 \\
Media & & & & & & & & \\
general & 45,65 & 0,05 & 0,54 & 3,00 & 16,14 & 3,90 & 0,74 & 3,46 \\
\hline
\end{tabular}

$\mathrm{DAP}=$ Diámetro a $1,3 \mathrm{~m}$.

$\mathrm{Vg}=$ varianza genotípica entre poblaciones. Ve= varianza residual o no explicada por el modelo. $\mathrm{Vf}=\mathrm{Va}+\mathrm{Vparc}+\mathrm{Ve}$ : varianza fenotípica total. $\mathrm{h}^{2} \mathrm{mf}=$ heredabilidad media de familia.

de $12,55 \mathrm{~m}^{3} / \mathrm{ha}(27,5 \%)$ con respecto al promedio del mismo ensayo. Si se compara el promedio de las dos procedencias derivadas colombianas con el de los materiales costarricenses (El Concho y Parrita), el diferencial de selección sería de $9,22 \mathrm{~m}^{3} /$ ha, que en términos de ganancia genética sería $8,76 \mathrm{~m}^{3} / \mathrm{ha}(20,3 \%)$.

Los resultados son consistentes, en los análisis realizados tanto a nivel de procedencias, progenies y árboles individuales, los materiales procedentes de Colombia demuestran superioridad en relación con los costarricenses, de Papúa Nueva Guinea y de República Dominicana; por lo que se debería continuar introduciendo material genético de este país para enriquecer el programa nacional con esta especie.

Finalmente, debe mencionarse que los resultados de esta investigación no deben ser extrapolados a un ambiente diferente al del sitio del ensayo (Zobel y Talbert 1988), ya que estos se basan en la expresión

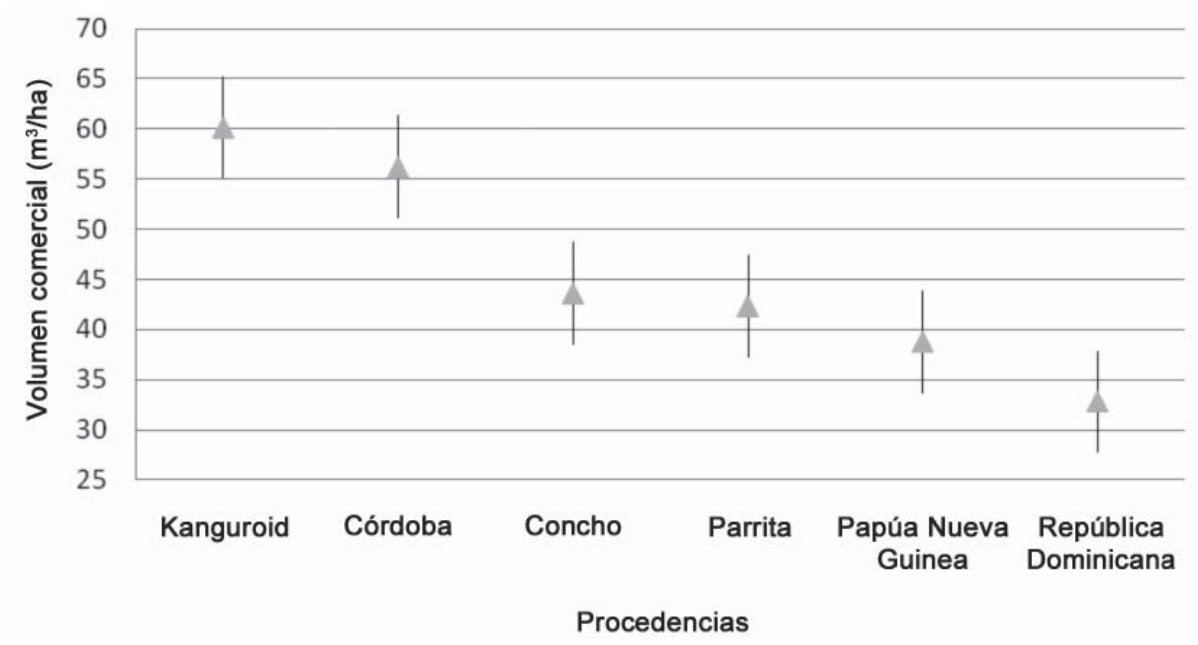

Figura 4. Valores genotípicos y límites de confianza para el Volumen Comercial por hectárea post raleo, al nivel de procedencias, en A. mangium de cuatro años de edad en Los Chiles, Costa Rica, 2010. 
Cuadro 9. Parámetros genéticos de seis procedencias de A. mangium evaluadas a los cuatro años de edad luego del raleo silvicultural. Los Chiles, Costa Rica, 2010.

\begin{tabular}{llcccc}
\hline Ranking & Procedencia & $\begin{array}{c}\text { Efecto genotípico } \\
\text { predicho }\end{array}$ & $\begin{array}{c}\text { Valor } \\
\text { genético }\end{array}$ & $\begin{array}{c}\text { Ganancia } \\
\text { esperada }(\mathbf{u + g})\end{array}$ & $\begin{array}{c}\text { Nueva } \\
\text { media }\left(\mathbf{m}^{3} / \mathbf{h a}\right)\end{array}$ \\
\hline 1 & Kanguroid & 14,54 & 60,19 & 14,54 & 60,19 \\
2 & Córdoba & 10,55 & 56,20 & 12,55 & 58,19 \\
3 & El Concho, San Carlos & $-2,04$ & 43,61 & 7,68 & 53,33 \\
4 & Parrita & $-3,33$ & 42,32 & 4,93 & 50,58 \\
5 & Papúa Nueva Guinea & $-6,88$ & 38,77 & 2,57 & 48,22 \\
6 & República Dominicana & $-12,85$ & 32,80 & 0,00 & 45,65 \\
\hline
\end{tabular}

(fenotipo) producto de los materiales genéticos utilizados en el ambiente seleccionado y no se determina el nivel de interacción genotipo-ambiente existente.

\section{LITERATURA CITADA}

Badilla, Y; Murillo, O; Obando, G. 2002. Efecto de la zona de vida y la altitud en la mortalidad y adaptabilidad al primer año de especies forestales en la Cordillera Volcánica Central, Costa Rica. Agronomía Costarricense 26(1):7-15.

CATIE (Centro Agronómico Tropical de Investigación y Enseñanza, CR). 1992. Mangium, Acacia mangium Will.: especie de árbol de uso múltiple en América Central. Turrialba, Costa Rica. CATIE. 62 p.

Espitia, M; Murillo, O; Castillo, C; Araméndiz, H; Paternina, N. 2010. Ganancia genética esperada en la selección de acacia (Acacia mangium WILLD) en Córdoba (Colombia). Revista U.D.C.A Actualidad y Divulgación Científica 13(2):99-107.

Guevara, M; Murillo, O. 2009. Costos y rendimientos de ocho tipos de poda en plantaciones jóvenes de Acacia mangium Willd en la zona norte de Costa Rica. Kurú 6(17):7.

Kim, N; Matsumura, J; Oda, K; Cuong, N. 2008. Possibility of improvement in fundamental properties of wood of acacia hybrids by artificial hybridization. Journal of Wood Science 55(1):8-12.

Murillo, O; Badilla, Y. 2004a. Breeding teak in Costa Rica. In IUFRO Meeting. Forest Genetics and Genomics. Charleston, South Carolina, USA. (en línea) Consultado 15 nov. 2011. Disponible en http://
www.ces.ncsu.edu/nreos/forest/feop/Agenda2004/ iufro_genetics2004/proceedings.pdf

Murillo, O; Badilla, Y. 2004b. Calidad y valoración de plantaciones forestales. Manual. Taller de Publicaciones del Instituto Tecnológico de Costa Rica. Escuela de Ingeniería Forestal. Cartago, Costa Rica. 51 p.

Murillo, O; Badilla, Y. 2006. Proyecciones de crecimiento de las plantaciones de Acacia mangium de ECOdirecta establecidas en la zona norte de Costa Rica. Informe de consultoría, FUNDATEC, octubre 2006. 27 p.

Murillo, O; Badilla, Y. 2011. Avalúos forestales (software). Instituto Tecnológico de Costa Rica. Cartago, Costa Rica.

Murillo, O; Obando, G; Badilla, Y; Araya, E. 2001.Estrategia de mejoramiento genético para el Programa de Conservación y Mejoramiento Genético de especies forestales del ITCR/FUNDECOR, Costa Rica. Revista Forestal Latinoamericana 16(30):273-285.

Nirsatmanto, A; Leksono, B; Kurinobu, S; Shiraishi, S. 2004. Realized genetic gain observed in secondgeneration seedling seed orchards of Acacia mangium in South Kalimantan, Indonesia. Journal of Forest Research 9(3):265-269.

Resende, MD V. de. 2002. Genética Biométrica e Estatística no Melhoramento de Plantas Perenes. EMBRAPA. Brasilia, Brasil. 975 p.

Resende, MD V. de. 2006. O Software Selegen-Reml/Blup. EMBRAPA. Campo Grande, Brasil. 299 p.

Resende,MD.V.de.2007.SELEGEN-REML/BLUP: Sistema Estatístico e Seleção Genética Computadorizada (Software). EMBRAPA. Brasilia, Brasil.

SIRZEE (Sistema de Información Regional - Zona Económica Especial). 2010. Mapas personalizados. (en línea). San Carlos, CR, ITCR. Consultado 5 jul. 2010. Disponible en http://www.sirzee.itcr.ac.cr 
Steel, R; Torrie, J. 1980. Principles and procedures of statistics. McGraw-Hill. New York, United States. $633 \mathrm{p}$.

van Bueren, M. 2004. Acacia hybrids in Vietnam: ACIAR Project FST/1986/030. ACIAR (Australian Centre for
International Agricultural Research). Canberra, AU. $44 \mathrm{p}$.

Zobel, B; Talbert, J. 1988. Técnicas de mejoramiento genético de árboles forestales. Editorial LIMUSA. México, D.F. México. 546 p. 